\title{
Hydrogen-assisted decohesion and localized plasticity in dual-phase steel
}

\author{
Motomichi Koyama $^{\text {a,c }}$, Cemal Cem Tasan ${ }^{\mathrm{a}, *}$, Eiji Akiyama ${ }^{\mathrm{b}}$, Kaneaki Tsuzaki ${ }^{\mathrm{b}, \mathrm{c}}$, \\ Dierk Raabe ${ }^{\mathrm{a}}$ \\ ${ }^{a}$ Max-Planck-Institut für Eisenforschung GmbH, Max-Planck-Straße 1, 40237 Düsseldorf, Germany \\ ${ }^{\mathrm{b}}$ National Institute for Materials Science, 1-2-1, Sengen, Tsukuba, Ibaraki 305-0047, Japan \\ ${ }^{\mathrm{c}}$ Department of Mechanical Engineering, Kyushu University, 744 Motooka, Nishi-ku, Fukuoka 819-0395, Japan
}

Received 22 November 2013; received in revised form 17 January 2014; accepted 19 January 2014

\begin{abstract}
Hydrogen embrittlement affects high-strength ferrite/martensite dual-phase (DP) steels. The associated micromechanisms which lead to failure have not been fully clarified yet. Here we present a quantitative micromechanical analysis of the microstructural damage phenomena in a model DP steel in the presence of hydrogen. A high-resolution scanning electron microscopy-based damage quantification technique has been employed to identify strain regimes where damage nucleation and damage growth take place, both with and without hydrogen precharging. The mechanisms corresponding to these regimes have been investigated by employing post-mortem electron channeling contrast imaging and electron backscatter diffraction analyses, as well as additional in situ deformation experiments. The results reveal that damage nucleation mechanism (i.e. martensite decohesion) and the damage growth mechanisms (e.g. interface decohesion) are both promoted by hydrogen, while the crack-arresting capability of the ferrite is significantly reduced. The observations are discussed on the basis of the hydrogen-enhanced decohesion and hydrogen-enhanced localized plasticity mechanisms. We discuss corresponding microstructure design strategies for better hydrogen-related damage tolerance of DP steels.
\end{abstract}

(c) 2014 Acta Materialia Inc. Published by Elsevier Ltd. All rights reserved.

Keywords: Hydrogen embrittlement; Dual-phase steel; Ferrite; Martensite; Damage

\section{Introduction}

Ferrite/martensite dual-phase (DP) steels are attractive materials for automotive applications [1-3], owing to a good combination of tensile strength (up to $1.2 \mathrm{GPa}$ ) and ductility (e.g. $10 \%$ or higher) $[1,4-6]$. This beneficial combination of mechanical properties in conjunction with a lean alloy concept is directly linked to the micromechanics of the composite-like ferrite-martensite microstructure. The high mechanical contrast inherent to such microstructures also introduces certain risk factors, namely, strong

\footnotetext{
* Corresponding author.

E-mail addresses: c.tasan@mpie.de, c.tasan@tue.nl (C.C. Tasan).
}

strain/stress partitioning [7-9], strain localization [10-12] and damage evolution, e.g. by martensite cracking $[1,5]$. While these micromechanical phenomena have been investigated in some detail, the associated hydrogen effects have not been accordingly addressed, despite reports on the susceptibility to hydrogen embrittlement (HE) of highstrength steels [13-15] and DP steels [1,16-19], and the increased susceptibility of ferrite and martensite phases to HE compared to stable austenite $[20,21]$. Our study aims to close this gap through a micromechanical analysis of the hydrogen-related microcracking mechanisms in DP steel.

HE in steels has been discussed mainly in terms of hydrogen-enhanced and strain-induced vacancies (HESIV) 
[22], hydrogen-enhanced decohesion (HEDE) [23-25] and hydrogen-enhanced localized plasticity (HELP) [26-29]. These embrittlement mechanisms, including their mutual interactions, are well documented in single-phase steels. For instance, the HE of fully ferritic steels was essentially ascribed to the HELP effect [30]. Lath martensitic steel, on the other hand, was reported to undergo HE associated with HELP as well as HEDE [31]. Given the more complex microstructures and local mechanics of DP steels, the connection of their HE to the HEDE, HESIV and HELP mechanisms, respectively, is expected to be more complex. In fact, indications of differences compared to single-phase materials were observed in a previous work on DP steels: unlike typical martensitic steels (which often show HE in the elastic or microplastic regimes [32-34]), HE of DP steels occurs after a significant level of plastic strain $[1,16,17]$. Unlike typical ferritic alloys, DP steels show significant microcracking (i.e. damage) before the final fracture even without hydrogen charging [5]. Note that this type of localized brittleness is caused by cracking of the martensite, i.e. the HE susceptibility of DP steel typically decreases by tempering [16] and increases with higher martensite fractions [17]. These observations underline the important influence of local plasticity and the damage sensitivity of the two phases on the overall HE susceptibility of DP steel. In order to elucidate these effects more clearly, we conduct here both (i) a quantitative assessment of the damage evolution and (ii) a crystallographic analysis of these microstructural phenomena in presence of hydrogen as a function of plastic strain.

Step-by-step quantitative analysis of micromechanical phenomena, such as gradual strain partitioning and damage evolution, are often not considered in conventional approaches to the evaluation of HE susceptibility of high-strength steels. Instead, certain critical macroscopic parameters (e.g. strain, external stress, stress intensity factor, hydrogen content) are typically determined when analyzing results obtained from flat $[33,34]$ or notched $[31,35,36]$ tensile samples. These more practical approaches are partially due to hydrogen-diffusion-related difficulties in experimentation, i.e. hydrogen can be released from the material, depending on the charging and environmental holding parameters. In order to render such analysis more quantitative and more microstructure-oriented, we investigate here the effects of hydrogen charging in conjunction with (i) electron backscatter diffraction (EBSD) based local misorientation measurements for assessing strain partitioning and (ii) a novel continuum damage mechanics-based approach to assess damage evolution, ${ }^{1}$ both conducted through post-mortem characterization of fractured samples, without any limitation due to hydrogen desorption.

\footnotetext{
${ }^{1}$ This method relies on determining permanent damage fraction (i.e. the ratio of the damaged to the undamaged cross-sectional area [37-39]) from high-resolution SEM imaging, and the strain levels corresponding to these damage values from digital image correlation (DIC).
}

A more detailed crystallographic and local plasticity-oriented analysis of the governing damage micromechanisms, on the other hand, requires the combined use of EBSD and electron channeling contrast imaging (ECCI) [40], as was conducted in previous works on 316 stainless steel and twinning-induced plasticity steel [41-43]. Here, the postmortem EBSD/ECCI technique is employed to retrace the hydrogen-assisted local plasticity and cracking mechanisms in DP steels. These analyses are also supported by in situ deformation experiments providing direct evidence of the observed mechanisms.

In the following, we first explain these experimental methods and the undeformed microstructures in detail. A full analysis of hydrogen-assisted damage evolution is then presented, introducing quantitative trends and the underlying micromechanisms. Finally, these results are discussed on the basis of the proposed HE theories.

\section{Experimental procedure}

A DP steel sheet with the chemical composition shown in Table 1 was solution treated at $1183 \mathrm{~K}$ for $1 \mathrm{~h}$, followed by air cooling to ambient temperature. The sheet was then intercritically annealed at $1023 \mathrm{~K}$ for $30 \mathrm{~min}$ and subsequently water quenched to produce a ferrite/martensite microstructure. Tensile specimens with gauge dimensions of $1.0 \mathrm{~mm}$ width $\times 0.4 \mathrm{~mm}$ thickness $\times 5.0 \mathrm{~mm}$ length were machined by mechanical grinding and spark erosion.

Hydrogen charging was performed for $1 \mathrm{~h}$ in a $5 \%$ $\mathrm{H}_{2} \mathrm{SO}_{4}$ aqueous solution containing $3 \mathrm{~g}^{-1}$ of $\mathrm{NH}_{4} \mathrm{SCN}$ at a cathodic current density of $2 \mathrm{~A} \mathrm{~m}^{-2}$. A platinum foil with dimensions of $25 \mathrm{~mm}$ width $\times 0.1 \mathrm{~mm}$ thickness $\times 25 \mathrm{~mm}$ length was used as the counter electrode. Since the surface area of the $\mathrm{Pt}$ foil in the solution was $1260 \mathrm{~mm}^{2}$, the anodic current density was about $0.8 \mathrm{~A} \mathrm{~m}^{-2}$. Since the effective diffusion coefficient of hydrogen is higher in ferrite than in martensite (i.e. due to the high density of dislocations in martensite acting as trapping sites, decreasing the diffusion speed), the diffusion coefficient of hydrogen in DP steels is considered to be higher than that in martensitic steels. The diffusion coefficient of hydrogen in martensite depends not only on the solute concentration but also on the tempering conditions [44-46]. Since the effective diffusion coefficient of hydrogen was reported to be $3.7 \times 10^{-11} \mathrm{~m}^{2} \mathrm{~s}^{-1}$ in an as-quenched low-alloy martensitic steel [45], the diffusivity of hydrogen in the DP steel is higher than the diffusivity of the martensitic steel. The hydrogen-affected zone can be estimated roughly by $(D t)^{1 / 2}$, where $D$ is effective diffusion coefficient of hydrogen and $t$ is the hydrogen-charging time. Assuming that the diffusion coefficient of hydrogen in the DP steel is $3.7 \times 10^{-11} \mathrm{~m}^{2} \mathrm{~s}^{-1}$, the hydrogen-affected zone just after hydrogen charging for $1 \mathrm{~h}$ is $0.365 \mathrm{~mm}$. Since hydrogen enters from both surfaces of the specimen, it can be safely claimed that the whole specimen was affected by hydrogen charging.

Thermal desorption analysis (TDA) was carried out from room temperature to $650 \mathrm{~K}$ using mass spectrometry 
Table 1

Chemical composition of the present steel.

\begin{tabular}{|c|c|c|c|c|c|c|c|c|}
\hline Alloy & $\mathrm{C}$ & $\mathrm{Mn}$ & $\mathrm{Si}$ & $\mathrm{Al}$ & $\mathrm{N}(\mathrm{ppm})$ & $\mathrm{P}$ & $\mathrm{S}$ & $\mathrm{Nb}$ \\
\hline wt. $\%$ & 0.147 & 1.709 & 0.249 & 0.040 & 39 & 0.011 & 0.004 & 0.011 \\
\hline $\mathrm{V}$ & $\mathrm{Ti}$ & $\mathrm{Cu}$ & $\mathrm{Sn}$ & $\mathrm{Cr}$ & $\mathrm{Ni}$ & Mo & $\mathrm{B}(\mathrm{ppm})$ & $\mathrm{Ca}(\mathrm{ppm})$ \\
\hline 0.003 & 0.003 & 0.011 & 0.002 & 0.543 & 0.022 & 0.002 & 5 & 23 \\
\hline
\end{tabular}

to measure the diffusible hydrogen content. The diffusible hydrogen, which is defined as the hydrogen that diffuses at ambient temperature, is reported to play a key role in HE [47]. The first TDA was started within 20 min after hydrogen charging under the same conditions as described above, and the second TDA was conducted after exposure in air at ambient temperature for 10 days. These measurements of hydrogen content were taken as the reference data in this study (although measurement inaccuracies can be introduced due to the $20 \mathrm{~min}$ duration [48]). The heating rate was $200 \mathrm{~K} \mathrm{~h}^{-1}$. A standard leak gas system of hydrogen using a crimper capillary whose hydrogen gas leak rate is $5.4 \times 10^{-11} \mathrm{~mol} \mathrm{~s}^{-1}$ was used for calibration. Calibration was done before each hydrogen desorption measurement. The diffusible hydrogen content was determined by measuring the cumulative desorbed hydrogen content from room temperature to $573 \mathrm{~K}$. Although various definitions of the diffusible hydrogen content are possible [49,50], the temperature range used in this study was determined for the following reasons: (i) the first peak finished at $573 \mathrm{~K}$; and (ii) the first peak disappeared after an exposure time of 10 days in air. The conventional TDA results in DP steels also showed that the first peak, corresponding to diffusible hydrogen, ends at around $573 \mathrm{~K}$ [1]. The diffusible hydrogen content was measured to be 0.66 mass ppm, and decreased to 0.02 mass ppm after the exposure to air for 10 days. The hydrogen desorption spectra are shown as Supplemental data.

The tensile tests were carried out along the rolling direction (RD) at an initial strain rate of $4.0 \times 10^{-3} \mathrm{~s}^{-1}$ at ambient temperature with and without hydrogen precharging. The tensile tests were conducted three times for each condition. In the case with hydrogen precharging, the tensile tests were started $20 \mathrm{~min}$ after the hydrogen charging had finished. In addition, an interrupted tensile test was conducted to investigate the hydrogen desorption-induced mechanical recovery. The specimen for this test was first deformed to $6 \%$ engineering strain with hydrogen precharging, and was subsequently unloaded. The deformed specimen was left in air at ambient temperature for 10 days to desorb most of the diffusible hydrogen, then deformed again until fracture.

Deformation experiments were conducted by using a Kammrath \& Weiss stage coupled with an Aramis system. Optical images, captured during the tensile tests, were postprocessed by digital image correlation (DIC) to measure the local strains on the tensile specimens [51,52]. A random pattern to obtain the local strains was provided by using a graphite spray. Quantitative values of deformationinduced damage were obtained by means of measurements of the damage area fraction, which is defined as $A_{d} / A_{a}$, where $A_{d}$ is the damaged cross-sectional area and $A_{a}$ is the area of the entire region observed. Since cracks and voids appear black in micrographs, the damaged area was measured from digitized scanning electron micrographs taken at various local plastic strains. The number of cracks per area was also measured from the same images. These measurements were conducted on the fractured specimens with and without hydrogen precharging.

Ex situ microstructure observations were performed by optical microscopy, secondary electron imaging, EBSD and ECCI to observe the initial microstructure consisting of ferrite and martensite and the deformation-induced cracks with and without hydrogen charging. The optical micrograph of the initial microstructure was taken after etching with a $10 \% \mathrm{HNO}_{3}+90 \% \mathrm{C}_{2} \mathrm{H}_{5} \mathrm{OH}$ solution and subsequent color etching with a $10 \% \quad \mathrm{Na}_{2} \mathrm{~S}_{2} \mathrm{O}_{5}$ aqueous solution on a mechanically polished surface. After the color etching, ferrite and martensite appear as white and black, respectively. The specimens for the scanning electron microscopic (SEM) observations were prepared by mechanical polishing with colloidal silica long enough to remove the layers affected by polishing with coarser particles. The SE and ECC imaging were operated at 20 and $15 \mathrm{kV}$, respectively. The EBSD analysis was operated at $15 \mathrm{kV}$, with a beam step size of $100 \mathrm{~nm}$.

In situ microstructure observations under tensile and three-point bending tests were conducted. The specimens for the in situ tests were mechanically polished and subsequently etched with a $10 \% \mathrm{HNO}_{3}+90 \% \mathrm{C}_{2} \mathrm{H}_{5} \mathrm{OH}$ solution. The ferrite and martensite phases in these observations were distinguishable as the ferrite phases are more sensitively etched compared to the martensite phases. We systematically studied etching effects in connection with EBSD measurements to improve the understanding on phase classification on etched surfaces. The in situ tensile test was employed to observe crack initiation and growth in a specimen without hydrogen charging. Since hydrogen desorption during observation is a critical issue for in situ SEM experiments, the in situ bending test was carried out under an optical microscope in a specimen that was precharged with hydrogen at a cathodic current density of $2 \mathrm{~A} \mathrm{~m}^{-2}$ for $1 \mathrm{~h}$. The in situ observation during the bending test was completed within $30 \mathrm{~min}$ after the hydrogen charging had finished. 

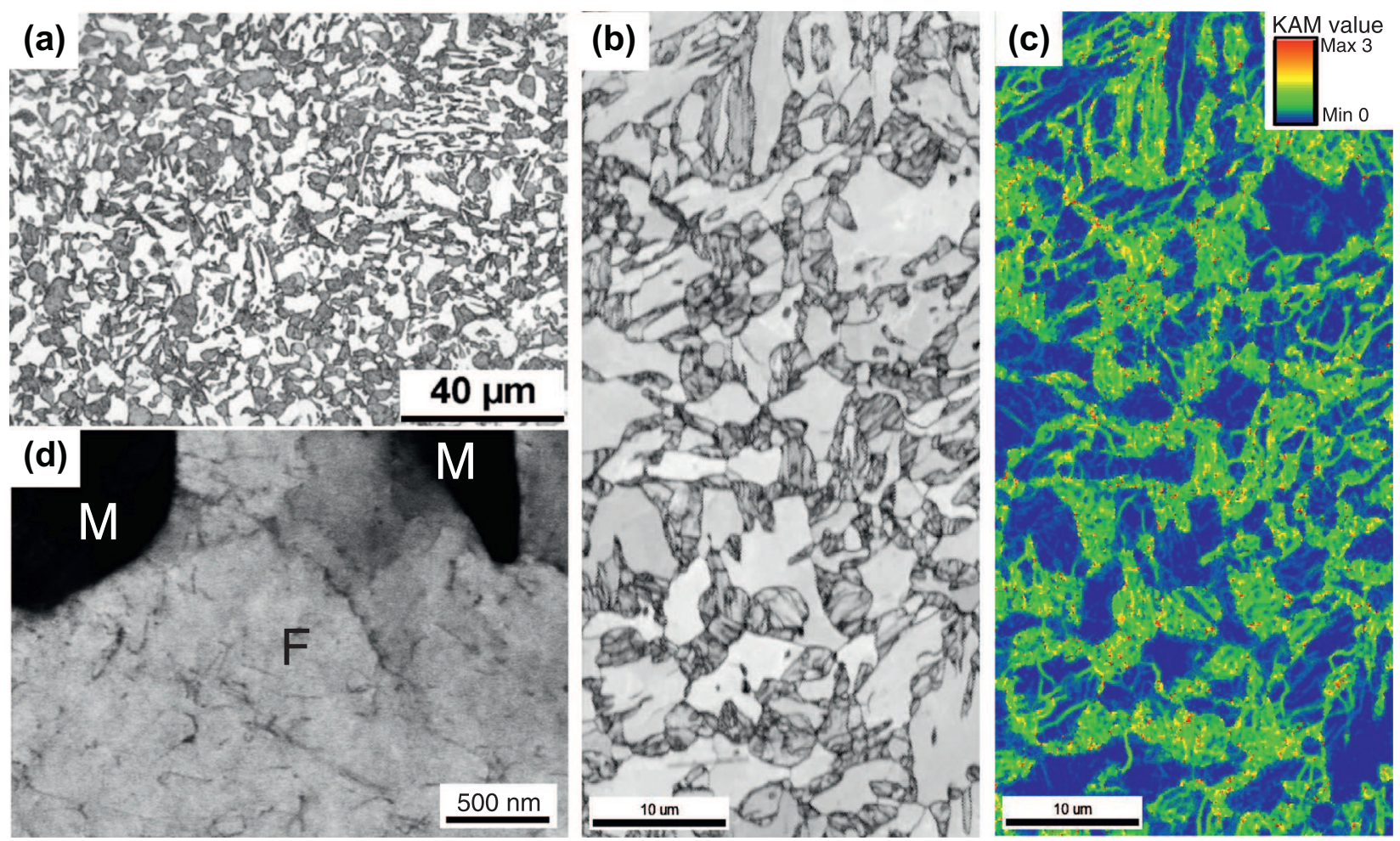

Fig. 1. Undeformed microstructures of the investigated DP steel. (a) Optical micrograph after the color etching, showing ferrite and martensite as white and black, respectively. (b) EBSD image quality map and (c) the corresponding KAM map. (d) Orientation-optimized ECC image. The KAM values were calculated using first neighboring EBSD points at a distance of $100 \mathrm{~nm}$. (For interpretation of the references to color in this figure legend, the reader is referred to the web version of this article.)

\section{Results}

\subsection{Characterization of the undeformed microstructure}

In Fig. 1a is presented an optical microscope image of the undeformed microstructure of the investigated steel, from which the martensite fraction is determined to be 55 vol.\%. The martensite distribution can also be shown by an EBSD image quality (IQ) map in Fig. 1b, where, due to the high density of lattice defects, martensite appears darker than ferrite [5]. Fig. 1c shows a map of the Kernel average misorientations (KAM), which quantifies the average misorientation around a measurement point with respect to a defined set of nearest neighbor points. KAM values have been shown to qualitatively correspond to the geometrically necessary dislocation density [5,53-55]. Therefore, the KAM map shown in Fig. 1c also visualizes martensite which contains a considerable number of lattice defects. Since hydrogen is trapped at lattice defects such as dislocations, it is assumed that the martensite contains a greater amount of hydrogen than the ferrite. Such a trend, i.e. an increase in hydrogen content with increasing lattice defect density, has been reported previously [56].

Note that the ferrite in the DP steel also contains a significant number of lattice defects even before deformation, especially near the phase boundaries (Fig. 1c) [55]. These dislocations are accommodating the martensitic transformation, as seem more clearly in the ECC image in Fig. $1 \mathrm{~d}^{2}$ Since these dislocations are also potential hydrogen trap sites, their presence suggests a heterogeneous distribution of diffusible hydrogen in ferrite, especially when comparing the vicinity of the phase boundaries with the grain interiors. The heterogeneity of hydrogen concentration, which depends on microstructure [57], is also an important factor for HE susceptibility.

\subsection{Hydrogen-assisted damage evolution: quantitative analysis}

The results of the tensile tests are presented in Fig. 2. The comparison of the results with and without hydrogen precharging clearly demonstrates the HE effect (Table 2). We observe that the yield and tensile strengths did not change significantly upon hydrogen charging, while the elongation to fracture deteriorated drastically. We note that the interrupted test shows a partial recovery of the elongation due to hydrogen desorption. The relevance of the observation will be discussed in Section 4.

\footnotetext{
${ }^{2}$ Since the surface orientation is optimized to reduce the electron backscattering yield, the original version of this ECC image showed dislocations with white contrast and the matrix with black contrast. Also, the martensite containing a considerable number of lattice defects is shown as white contrast. Here we inverted the image for visual clarity in the print version.
} 


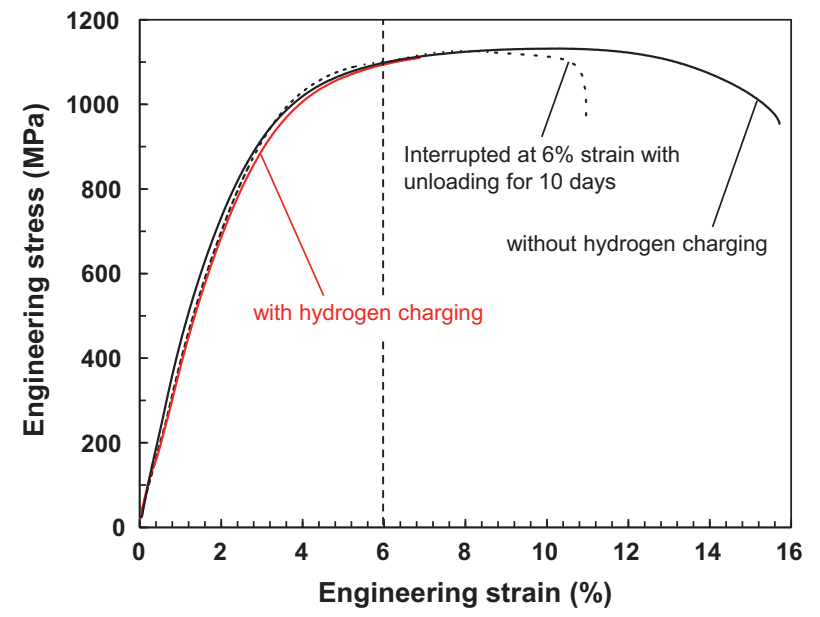

Fig. 2. Engineering stress-strain curves of the specimens with and without hydrogen precharging. In addition, the result of the interrupted tensile test is superimposed on two of the curves. In the interrupted test, hydrogen was introduced before the tensile test, and the test was stopped at $6 \%$ strain and subsequently unloaded. After exposure to air for 10 days, the specimen was reloaded to fracture.

Table 2

Tensile mechanical properties.

\begin{tabular}{llll}
\hline & $0.2 \%$ PS $(\mathrm{MPa})$ & UTS $(\mathrm{MPa})$ & Total elongation $(\%)$ \\
\hline Without H & $789 \pm 27$ & $1168 \pm 69$ & $13.0 \pm 1.7$ \\
With H & $819 \pm 27$ & $1148 \pm 71$ & $6.3 \pm 0.6$ \\
\hline
\end{tabular}

The tensile tests were conducted three times. PS: proof stress; UTS: ultimate tensile strength.

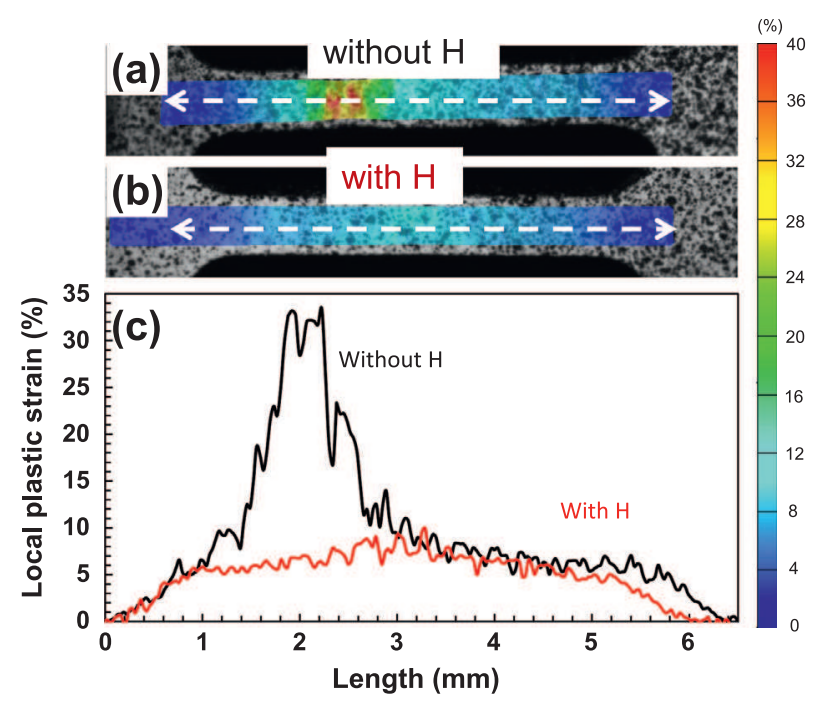

Fig. 3. DIC strain map on the set of failure in the specimens deformed (a) without hydrogen charging (total elongation: $12 \%$ ) and (b) with hydrogen charging (total elongation: 6\%). (c) Local plastic strain along the profiles in (a) and (b). Note that the local plastic strains are almost identical except for the necking region.

When comparing the local plastic strain distributions of the specimens just before the fracture with and without hydrogen precharging using the DIC analyses (Fig. 3), we find that the local plastic strain is almost identical in the two samples except for the necking region. This means that hydrogen does not affect the uniform elongation regime but causes the ductility to deteriorate specifically in the postnecking regime. Hence, a more detailed inspection of the hydrogen effects is conducted in the necking regime.

We next concentrate on the influence of hydrogen on the deformation-induced damage evolution. Fig. 4 shows the evolution of the damage area fraction, the number of damage incidents per area and the average crack size, all as a function of the applied nominal strain. The average crack size, $d_{\text {ave }}$, is calculated from the prior two values, i.e. as $d_{\text {ave }}=D_{a} / n$, where $D_{a}$ is the damage area fraction and $n$ the number of cracks per area. Examining Fig. 4c as an example without hydrogen charging, the damage evolution can be classified into three stages: (i) damage incubation $(0-9 \%)$; (ii) damage nucleation (9-30\%); and (iii) damage growth $(30 \%$-fracture), where the percentage refers to the local strain. The average crack size was roughly constant in the damage nucleation regime. In the case of the hydrogen-charged specimen, we observed that all three damage evolution stages were significantly reduced to much smaller strains by the hydrogen uptake. We also find that the average crack size $\left(\sim 0.35 \mu \mathrm{m}^{2}\right)$ during the damage nucleation stage was not changed by the hydrogen charging.

\subsection{Hydrogen-assisted damage evolution: underlying micromechanisms}

Fig. 5a-c shows results obtained from the in situ SEM tensile test conducted on the specimen which was not hydrogen-charged. Fig. $5 \mathrm{a}^{\prime}-\mathrm{c}^{\prime}$ shows magnified images corresponding to the regions outlined by the white broken lines. These observations were initiated after necking was confirmed. Since Fig. 5a was obtained after the necking and just before the engineering stress decrease $(11 \%$ macroscopic strain), this corresponds to the late damage nucleation regime. The micrograph from the late damage nucleation regime identifies martensite cracking as the dominant mechanism (see the red arrows in Fig. 5a and $\left.\mathrm{a}^{\prime}\right)$. With further deformation, the damage size increased, yet the crack propagation paths were within the martensite or along the ferrite/martensite interfaces (see the yellow arrows in Fig. 5b, c, b $\mathrm{b}^{\prime}$ and $\mathrm{c}^{\prime}$ ). The crack propagation paths were never observed inside the ferrite grains even in the vicinity of the fracture surface. Previous studies on the damage evolution of DP steel without hydrogen charging also showed such martensite and ferrite/martensite interface decohesion mechanisms [5,7,58].

We next examine damage nucleation in the presence of hydrogen at various local plastic strain levels in the hydrogen-charged specimen, as shown in Fig. 6. Fig. 6a shows a crack that was observed in the early damage nucleation regime $(\sim 2 \%$ local plastic strain). Similarly to the case without hydrogen charging, the crack was initiated in the martensite region (Fig. 6b). At 5\% local plastic strain, new damage incidents are nucleated (see red arrows in 

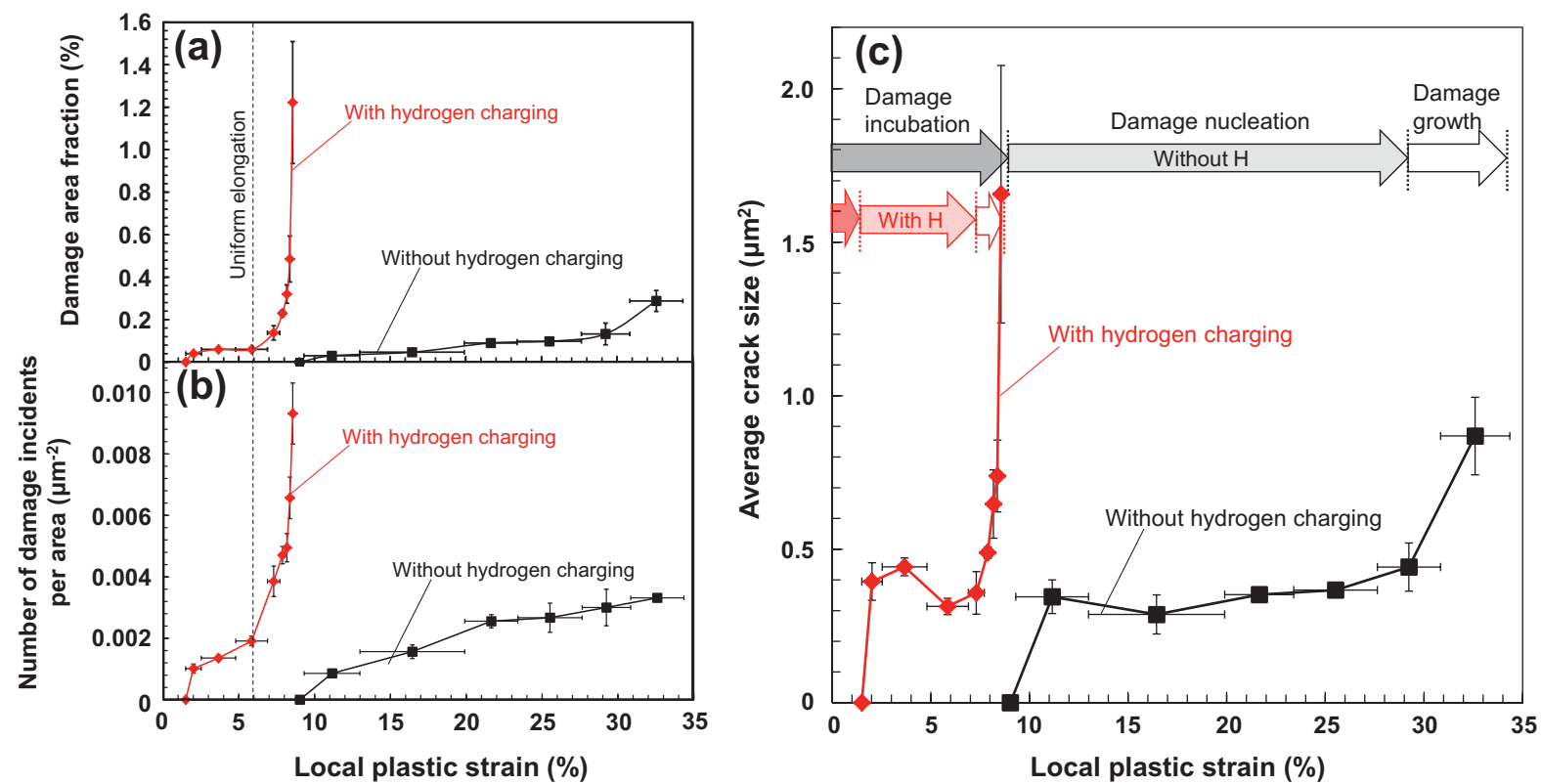

Fig. 4. Damage evolution curves plotted against local plastic strain with and without hydrogen charging: (a) damage area fraction, (b) number of damage incidents per area and (c) average crack size. The total elongations of the used specimens with and without hydrogen were $6 \%$ and $12 \%$, respectively.

\section{Damage nucleation regime}
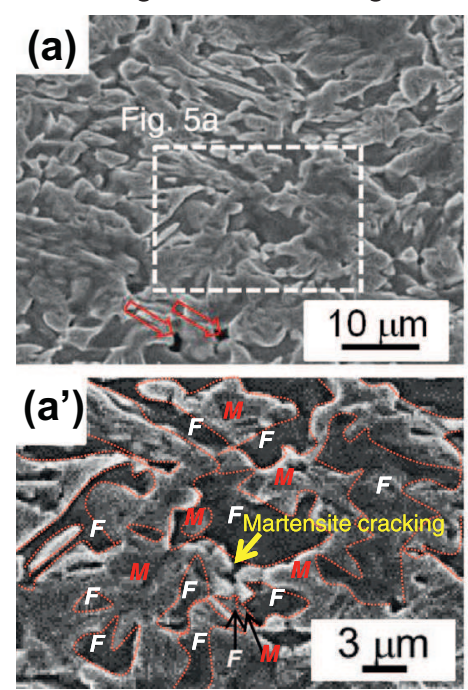

\section{Damage growth regime}

(b)
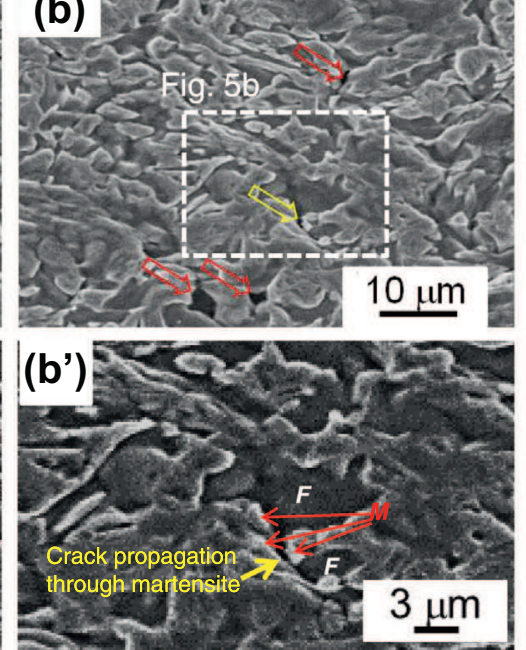

Damage growth regime (fracture)
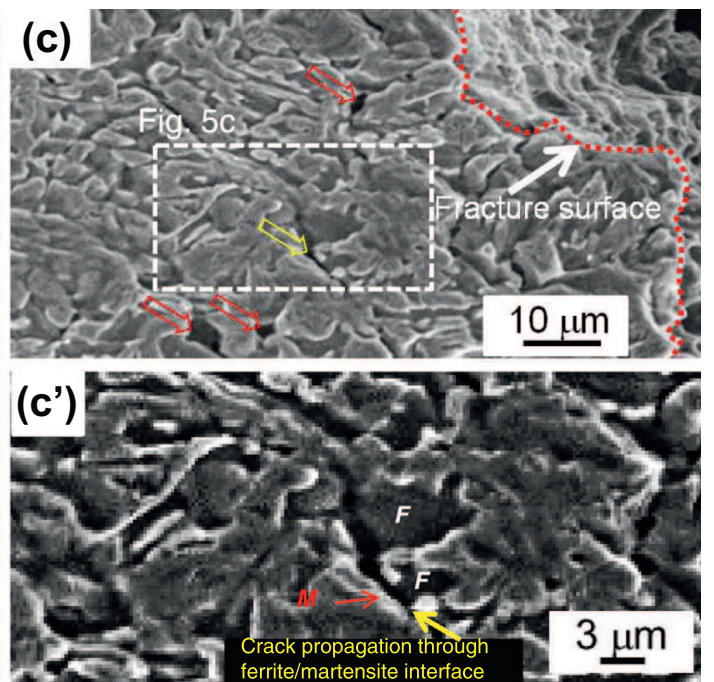

Fig. 5. In situ SEM observations during tensile testing without hydrogen charging in (a) the damage nucleation regime (11\% macroscopic strain), (b) the damage growth regime ( $12 \%$ macroscopic strain) and (c) the late damage growth regime (at the vicinity of the fractured part). The strains are macroscopic strain, not local plastic strain. The dotted red lines in (c) indicate the fracture surface. According to Fig. 3, the local strain in the vicinity of fracture surface is speculated to be about $34 \%$ plastic strain. $\left(a^{\prime}-c^{\prime}\right)$ The magnified images corresponding to the regions outlined in $(a-c)$. The red dotted lines in $\left(a^{\prime}\right)$ indicates ferrite $(\mathrm{F}) /$ martensite $(\mathrm{M})$ interfaces. (For interpretation of the references to color in this figure legend, the reader is referred to the web version of this article.)

Fig. 6c), yet, even when the new cracks are nucleated in the vicinity of a pre-existing crack, the cracks did not coalesce, but instead remain confined inside the martensite regions (Fig. 6d). Only in the late damage nucleation regime ( $\sim 7 \%$ local plastic strain) did the martensite cracks start to propagate through a few grains (see the red arrows in Fig. $6 \mathrm{e}$ and the corresponding magnified image in Fig. 6f). In the late damage growth regime $(\sim 10 \%$ local plastic strain), a considerable amount of well-developed damage incidents were observed (Fig. 6g). However, most cracks propagated only through a few grains and, interestingly, many cracks grew only along the tensile direction, without showing coalescence through crack growth in thickness and width directions. These facts clearly indicate that the hydrogen-enhanced damage evolution in the damage growth stage is not caused by coalescence of hydrogenrelated martensite cracks or by the simple interface damage mechanism that was observed in hydrogen-free samples. 


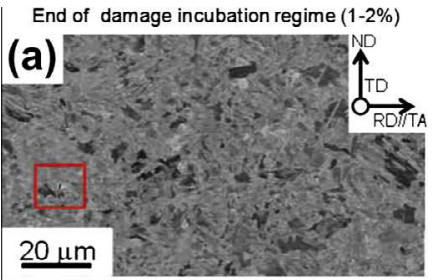

Early damage nucleation regime $(4-5 \%)$
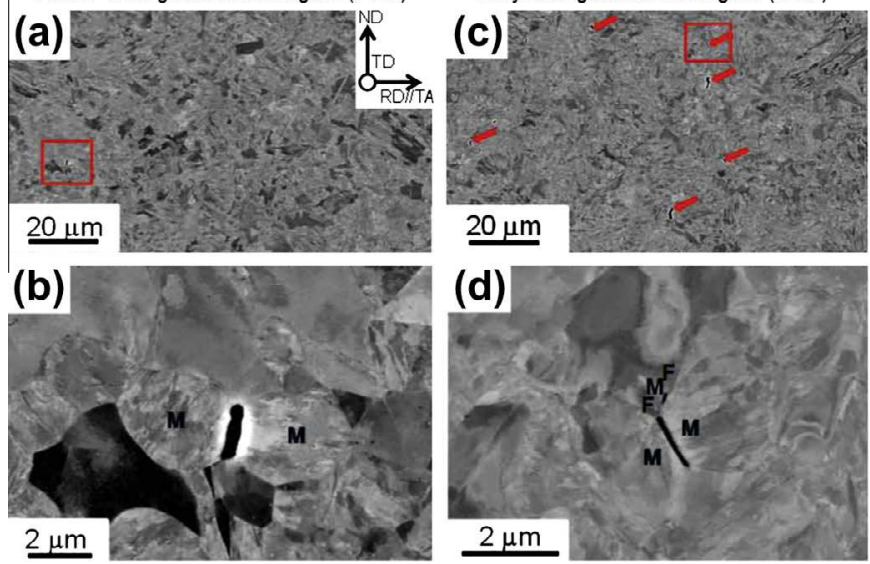
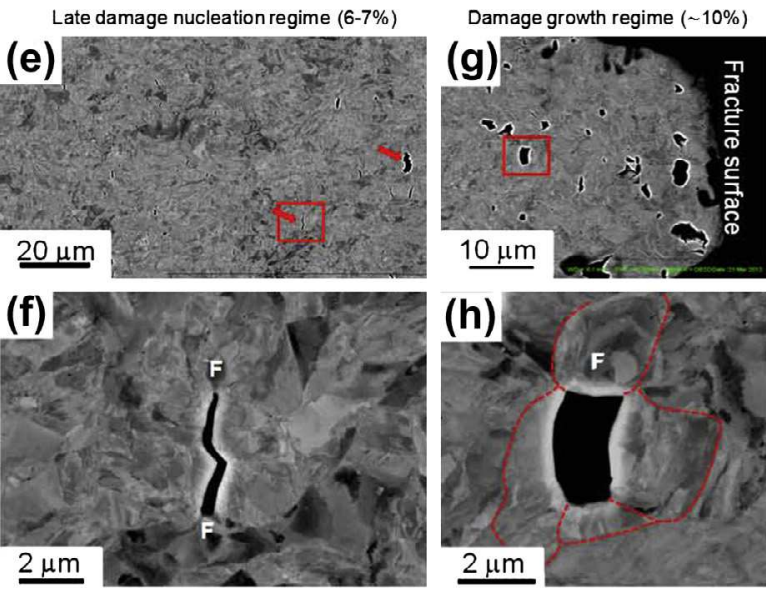

Fig. 6. ECC images at two different magnifications showing damage evolution as a function of plastic strain in the fractured specimen with hydrogen precharging in $(\mathrm{a}, \mathrm{b})$ the end of the damage incubation regime (1-2\% local plastic strain), (c, d) the early damage nucleation regime (4-5 local plastic strain), (e, f) the late damage nucleation regime (6-7\% local plastic strain) and $(\mathrm{g}, \mathrm{h})$ the damage growth regime $(\sim 10 \%)$. The magnified images correspond to the squares in the lower magnification image. Some of the cracks are indicated by the red arrows. The broken red line in (h) indicates prior austenite grain or ferrite/martensite phase boundaries around the crack. F and $\mathrm{M}$ indicate ferrite and martensite, respectively. The total elongations of the used specimens with and without hydrogen were $6 \%$ and $12 \%$, respectively. (For interpretation of the references to color in this figure legend, the reader is referred to the web version of this article.)

Comparing the fracture behavior in DP steel with and without hydrogen charging also reveals another interesting fact (Fig. 7a and b): the fracture surfaces of both types of specimens show dimples as well as brittle features, as indicated by red dotted lines. The fracture surface of the interrupted test also exhibits a similar feature, as shown in Fig. 7c (the relevance of this fractograph will be discussed in Section 4). Although the fractographs were taken in a vicinity of the edge, these features were observed also in the central region of the specimens. The appearance of dimples indicates that coalescence of voids and cracks contributed to the final fracture. Since the size of each brittle region is larger than the grain size (shown in Fig. 1a), the brittle fracture regions of both types of specimens result from brittle crack propagation through multiple grains. The hydrogen charging increased the size of the brittle fracture region, and the large brittle feature region contains a considerable number of voids and dimples, as indicated by the red arrows in Fig. 7b. The martensite-related cracking associated with decohesion is considered to be the cause of the partial brittle fracture surface. The voids and dimples observed in the hydrogen-charged sample indicates that hydrogen-assisted crack propagation was caused by decohesion as well as ductile cracking. In addition, the final fracture in both the hydrogen-charged and the hydrogenfree samples was caused not only by brittle cracking but also by coalescence of microvoids, since the fracture surface includes many dimples, as shown in Fig. 7a and b.

To understand the micromechanics behind these observations, various EBSD measurements were carried out in the damage nucleation and growth regimes of the same specimens used for the ECCI observations shown in Fig. 6. We first focus on the martensite cracking, observed in all samples in the damage nucleation regime, to better understand the assistance of hydrogen in reducing the damage incubation regime. Fig. 8a shows an IQ map at $2 \%$ local plastic strain for the hydrogen-charged sample. Cracks were observed here in the martensite region, as shown in Fig. 6b. Fig. 8b shows the magnified image of the part outlined by the square in Fig. 8a. This image more clearly demonstrates that the cracking occurred inside the martensite. The RD inverse pole figure (IPF) map shown in Fig. $8 \mathrm{c}$ indicates that the crack has propagated along the prior austenite grain boundary, which was analyzed from the crystallographic orientations. Namely, the misorientations between neighboring grains on the crack did not correspond to the conventionally reported values for packet/block/lath boundaries [59], and the crack did not run along the $\{110\}$ planes, on which the lath boundaries lie [34]. Hydrogen was reported to exist on prior austenite grain boundaries preferentially in martensite [60], which strongly suggests that the preferential prior austenite grain boundary cracking observed at small strains is caused by enhanced hydrogen concentrations at such interfaces.

It is also important to note that the prior austenite grain boundary cracking was arrested at the surrounding ferrite grains by localized plasticity in the ferrite (see the KAM map shown in Fig. 8d). Fig. 8e schematically describes the cracking mechanism described above. A sharp crack is first initiated on the prior austenite grain boundary, and is then arrested by plastic deformation of the ferrite grains around the crack tip, resulting in crack blunting, as shown in Fig. 8b.

With further deformation in the damage nucleation regime, ferrite/martensite interface cracking and block/lath boundary cracking mechanisms are also observed, as well as prior austenite boundary cracking (see Fig. 8f showing an SE image of the $70^{\circ}$-tilted specimen with $7 \%$ local plastic strain). For the former, the corresponding IQ map shown in Fig. $8 \mathrm{~g}$ enables identifications of ferrite and martensite, 

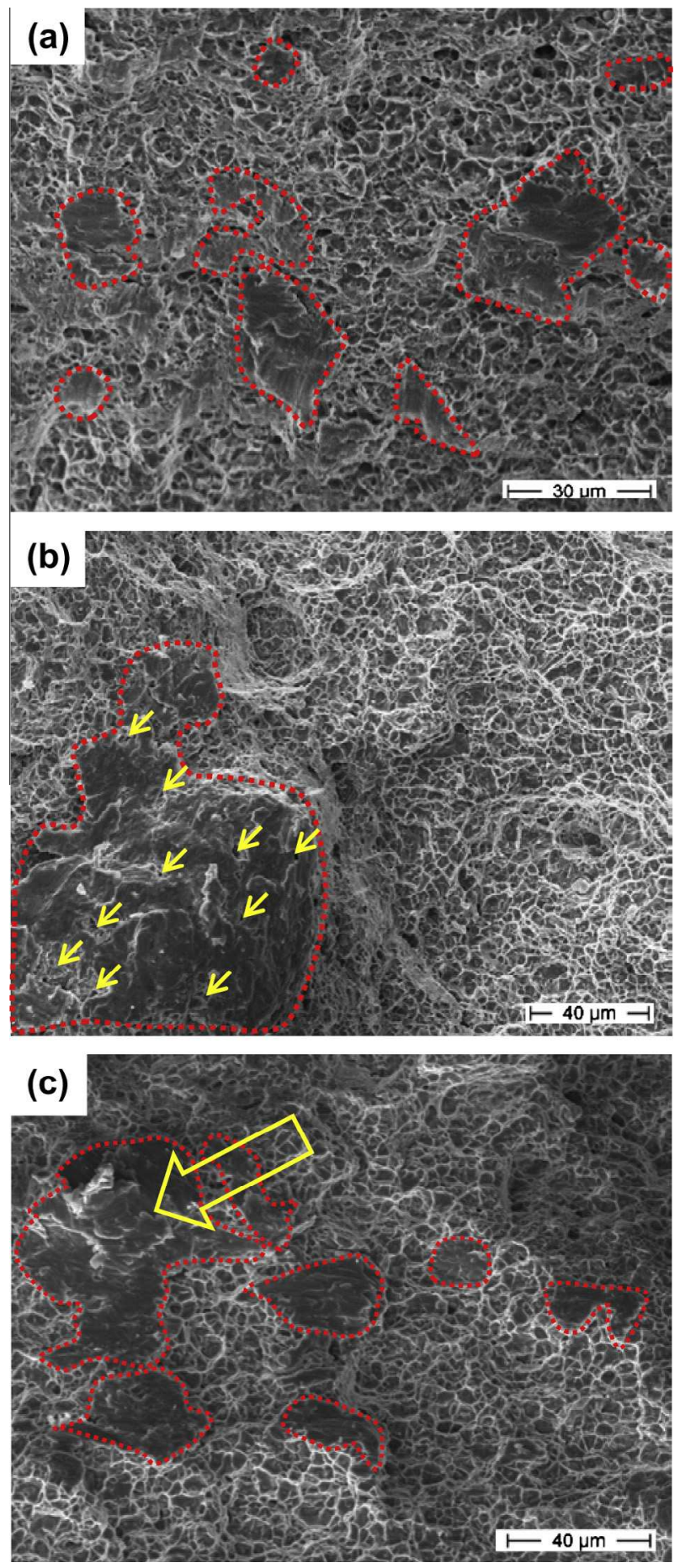

Fig. 7. Fracture surfaces of the specimen (a) without and (b) with hydrogen charging. The red dotted lines indicate brittle fractured regions. The total elongations of the used specimens with and without hydrogen were $6 \%$ and $12 \%$, respectively. (c) Fracture surface of the specimen for the interrupted test shown in Fig. 2. (For interpretation of the references to color in this figure legend, the reader is referred to the web version of this article.)

and for the latter, based on the specific crystallographic relationship on lath martensite [59], the RD IPF map shown in Fig. 8h allows identifications of block/lath boundary cracking and prior austenite grain boundary cracking, respectively. The cracks observed here that are aligned along the block/lath boundaries match crystallographic $\{110\}_{\mathrm{M}}$ planes, similar to the observations previously reported on quasi-cleavage cracking of martensitic steels $[34,61,62]$. Note also that prior austenite grain boundary cracking was reported in fully martensitic steel as well [34], indicating that the hydrogen-assisted martensite cracking in DP steels has similarities with that in fully martensitic steels.

From these observations, it can be concluded that the reduction in the critical strains required for martensite cracking and ferrite/martensite cracking is caused by hydrogen localizing at certain boundaries and its assistance in cracking. The observation of martensite cracking and ferrite/martensite interface cracking in the hydrogen-free specimen (Fig. 5) also supports the hypothesis that hydrogen assists rather than fully induces these mechanisms.

When aiming to reconstruct how this process takes place, it should be underlined that the decohesion behavior in DP steels depends on the cohesive energy of the martensite-related boundary (i.e. prior austenite grain boundary, block/lath boundary and ferrite/martensite interface boundary) and the number of dislocations piling up at the boundaries [55]. In a previous work, the correlation between dislocation pile-ups and decohesion was clarified by a KAM analysis to explain suppression of decohesion by grain refinement in a DP steel [5]. Fig. 9 shows the KAM distribution in ferrite and martensite of the undeformed and deformed specimens with and without hydrogen precharging. The data taken at $7 \%$ local plastic strain reveal that the peak of the KAM distribution broadens and shifts to higher values particularly for ferrite in case of both types of samples, i.e. hydrogen-charged and hydrogen-free specimens. However, the KAM distribution does not show a significant difference between the specimens with and without hydrogen charging even at $7 \%$ local plastic strain. This clearly suggests that the main cause of the hydrogen-assisted martensite cracking and ferrite/ martensite cracking is the influence of hydrogen not on the dislocation pile-ups but, rather, directly on the cohesive interface energy. The promotion of brittle cracking associated with the reduction in cohesive energy is described by the so-called HEDE mechanism.

We next discuss the damage growth regime in Fig. 4c: Fig. 10 shows typical crack propagation characteristics discovered in this regime through high-resolution EBSD measurements. The IQ map presented in Fig. 10a allows the identification of ferrite and martensite grains. We observe that the crack propagation has taken place in the interior of the ferrite grain surrounding the martensite islands. Considering the significant difference introduced by the presence of hydrogen to the critical local plastic strain level when the damage growth regime is initiated, we propose that hydrogen has a significant effect on the further propagation of cracks, formed inside the martensite regions, into the soft surrounding ferrite grains. When analyzing the RD IPF and KAM maps in Fig. $10 \mathrm{~b}$ and $\mathrm{c}$, it is seen that the 
End of damage incubation regime (1-2\%) Late damage nucleation regime (6-7\%)

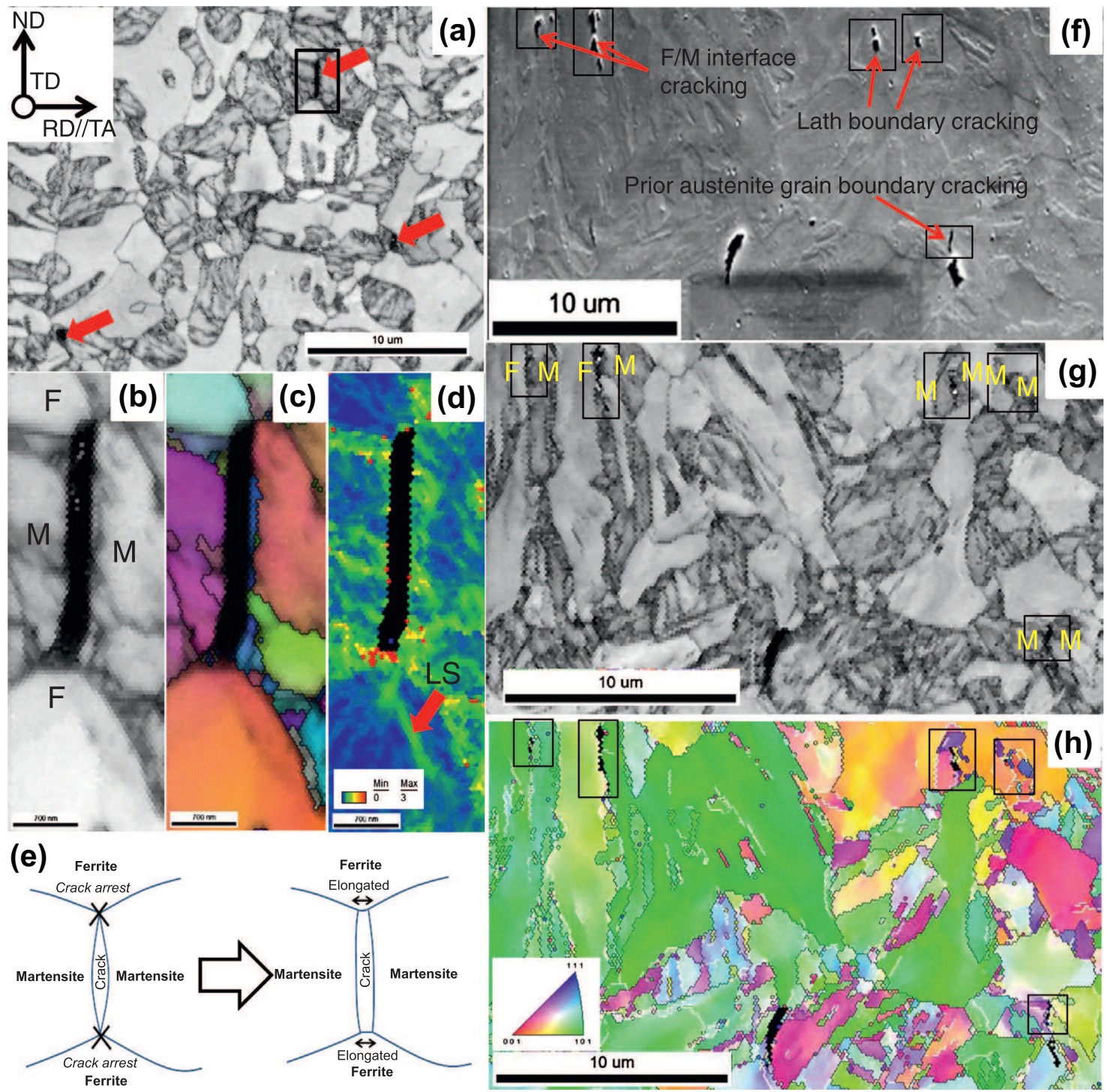

Fig. 8. (a) IQ map at a 1-2\% local plastic strain (end of the damage incubation regime). The red arrows indicate cracks. (b) IQ, (c) RD IPF and (d) KAM maps corresponding to the part surrounded by the square in (a). (e) Schematics for the crack initiation and arrest process. (f) SEM image of the specimen tilted to $70^{\circ}$ at $6-7 \%$ local plastic strain (late damage nucleation regime). (g) IQ and (h) RD IPF maps corresponding to the SEM image in (f). The parts highlighted by the squares in $(\mathrm{d}-\mathrm{h})$ show cracks, and correspond to each other across the images. All of these images were taken on the fractured specimen with hydrogen precharging. F: ferrite, M: martensite, LS: localized slip. (For interpretation of the references to color in this figure legend, the reader is referred to the web version of this article.)

local orientation changes and high KAM values occur around the crack tip. This observation underlines a particular characteristic and function of the ferrite phase in DP steels, namely, its crack-arresting capability. The localized slip activity at the crack tip observed in Fig. 10c proves that this ability is still present under the presence of hydrogen. However, when considering the drop in the critical strain where the crack growth regime is initiated (Fig. 4c), we propose that hydrogen strongly decreases the crack-arresting property of ferrite. This effect is considered to arise from hydrogen localization in the vicinity of the crack tip and the subsequent hydrogen-assisted easing of localized plastic shear in ferrite.
Considering these points, the details of the crack opening process observed in Fig. 10a-c can be schematically described, as shown in Fig. 10d-h, to provide a generalized overview of the micromechanics responsible of the damage growth regime in Fig. 4. The crack is initiated in the martensite region (Fig. 10d) and then propagates along the ferrite/martensite interface (Fig. 10e), in accordance with the mechanism discussed with Fig. 8 (and depicted schematically in Fig. 8e). Note that this cracking process was observed even without hydrogen precharging, as shown in Fig. 5. Such cracks are initially (i.e. in the damage nucleation regime) arrested when abutting the surrounding ferrite grains, as shown by the strain localization inside 

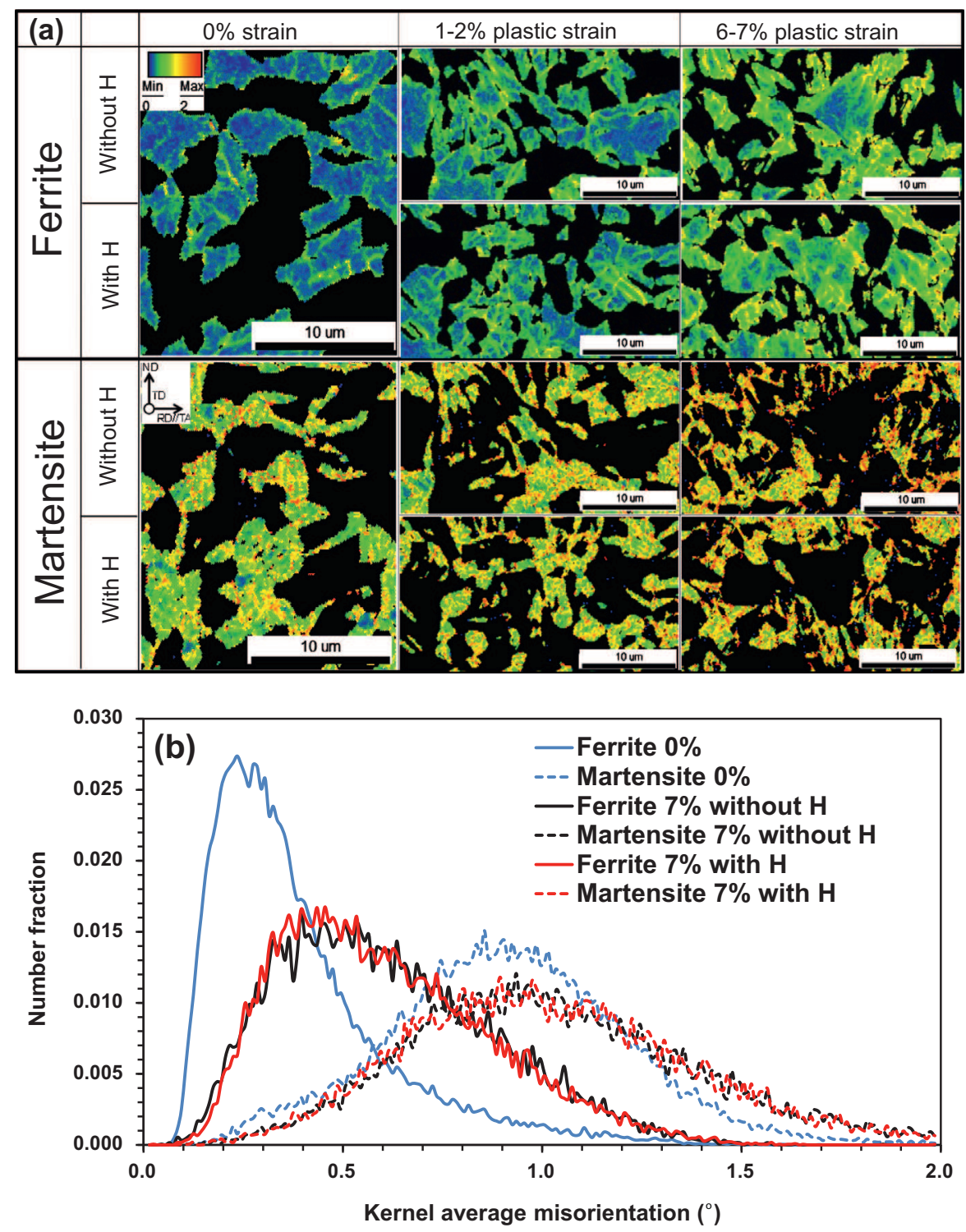

Fig. 9. (a) KAM maps at different plastic strain levels with and without hydrogen charging. The ferrite and martensite were separated in terms of grain average IQ value. (b) KAM value distribution of the specimen with and without hydrogen charging at $0 \%$ and $7 \%$ local plastic strains. The condition for the calculation of KAM values is the same with Fig. 1.

the ferrite in Fig. 8d. Considering also the stress field at the crack tip along with the strain localization, hydrogen localization in the vicinity of the crack tip is an expected consequence, ${ }^{3}$ resulting in the hydrogen-assisted crack propagation into ferrite (Fig. 10f). The crack is arrested by the further plastic deformation in ferrite, shown in terms of the local orientation change and locally high KAM values. When the crack has opened sufficiently wide along the tensile direction by tearing the plastically deformed ferrite region (Fig. 10g), the crack is finally arrested completely,

\footnotetext{
${ }^{3}$ For instance, hydrogen that exists in interstitial sites diffuses even in the absence of a stress field up to $\sim 180 \mu \mathrm{m}$ within $30 \mathrm{~s}$, based on the assumption of $(D t)^{1 / 2}$ in ferrite of an annealed mild steel (where $D$ is $\left.1.05 \times 10^{-9} \mathrm{~m}^{2} \mathrm{~s}^{-1}[45]\right)$. The stress gradient also promotes the hydrogen diffusion to the crack tip.
}

due to the local plastic deformation of the ferrite grain (Fig. 10h) and the associated reduction in the local stress intensity factor (crack blunting effect). As a result of the process summarized in Fig. $10 \mathrm{~d}-\mathrm{h}$, the wide-open, blunt crack shown in Fig. 10a-c is produced. Hence, in the presence of hydrogen, the damage growth regime (Fig. 5c) is dominated by ferrite/martensite interface cracking and ferrite cracking.

To confirm the mechanisms identified by post-mortem analyses presented above, in situ light microscopy bending tests were carried out after hydrogen charging (see Fig. 11 and the Supplementary video file). Although the initial bending step did not show any cracking (Fig. 11b), martensite cracking is observed in the following step, as indicated by the red arrow (Fig. 11c) and the dotted circle (Fig. 11d). The crack propagated into the ferrite grain, as 

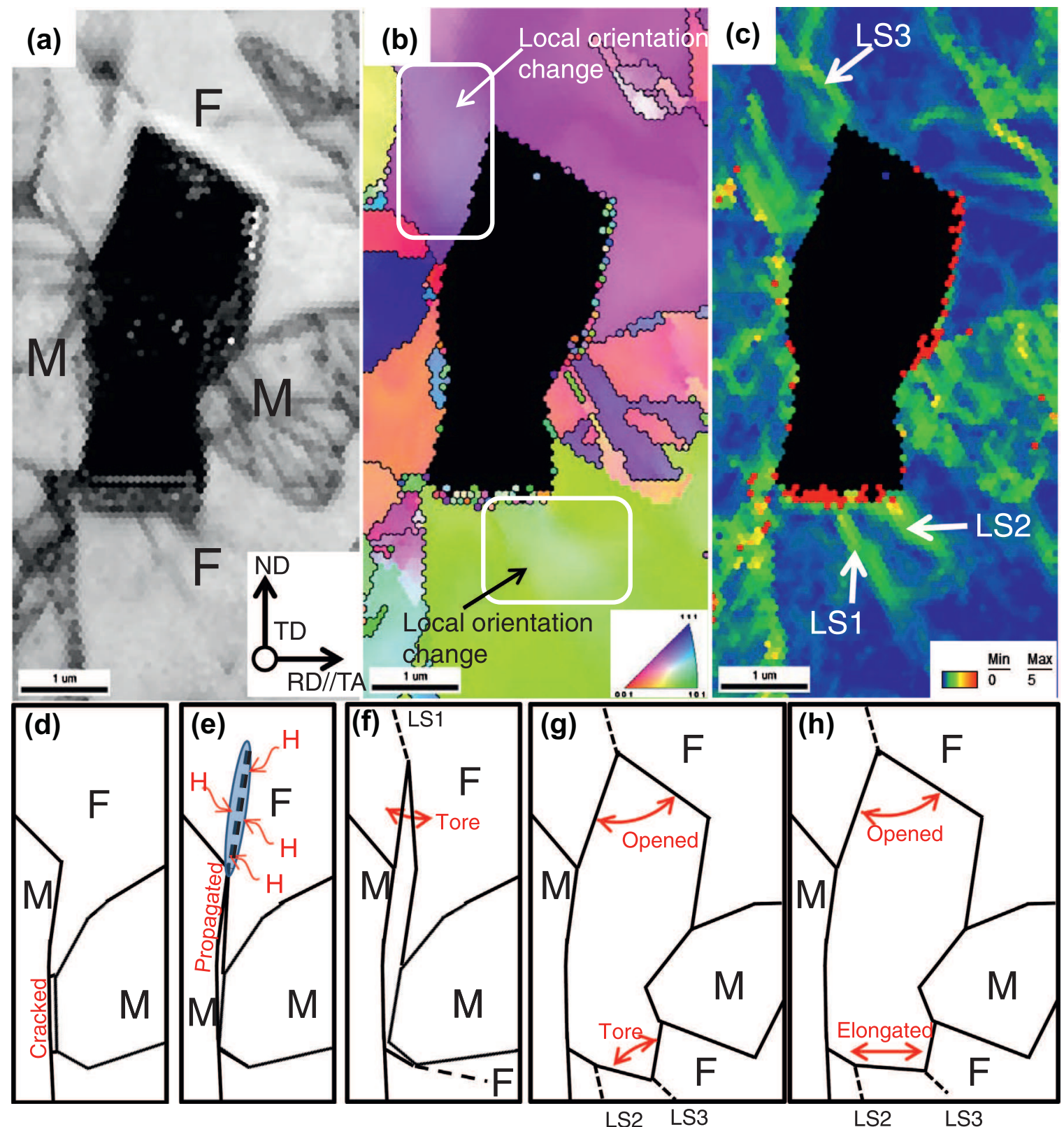

Fig. 10. (a) IQ, (b) RD IPF and (c) KAM maps at 7\% local plastic strain of the sample with hydrogen precharging. (d-h) Schematics demonstrating how the crack shown in $(\mathrm{a}-\mathrm{c})$ nucleated, propagated and opened. F: ferrite, M: martensite, LS: localized slip.

indicated by the dotted circle (Fig. 11e). The crack propagation and further deformation opened the crack (Fig. 11f). This crack-opening process is considered to correspond to Fig. 10. These observations indicate that the well-opened cracks shown in Fig. $6 \mathrm{~g}$ and $\mathrm{h}$ that occur in the presence of hydrogen are attributed to ferrite/martensite interface cracking and crack propagation from the interface into the ferrite, as well as ferrite/martensite boundary sliding (the boundary sliding can be observed more clearly in the Supplementary video file). The latter mechanism can be observed during the bending, as shown in Fig. 11c-f, where the cracked martensite, indicated by the red arrow, slides along the ferrite/martensite interface.

\section{Discussion: the role of the HEDE and HELP mechanisms in hydrogen-assisted fracture of DP steel}

Here we discuss the observed hydrogen effects on cracking in the context of the HEDE and HELP mechanisms, which are based on the reduction in cohesive energy and on the increase in dislocation mobility, respectively. As mentioned in Section 3.2, the damage evolution curves were separated into the three subsequent stages in terms of the critical strains observed for damage nucleation and for the onset of damage growth. As shown in Figs. 5, 6a and $\mathrm{b}$ and $8 \mathrm{~b}$ and $\mathrm{c}$, the critical strain for damage nucleation was controlled by martensite cracking, a 

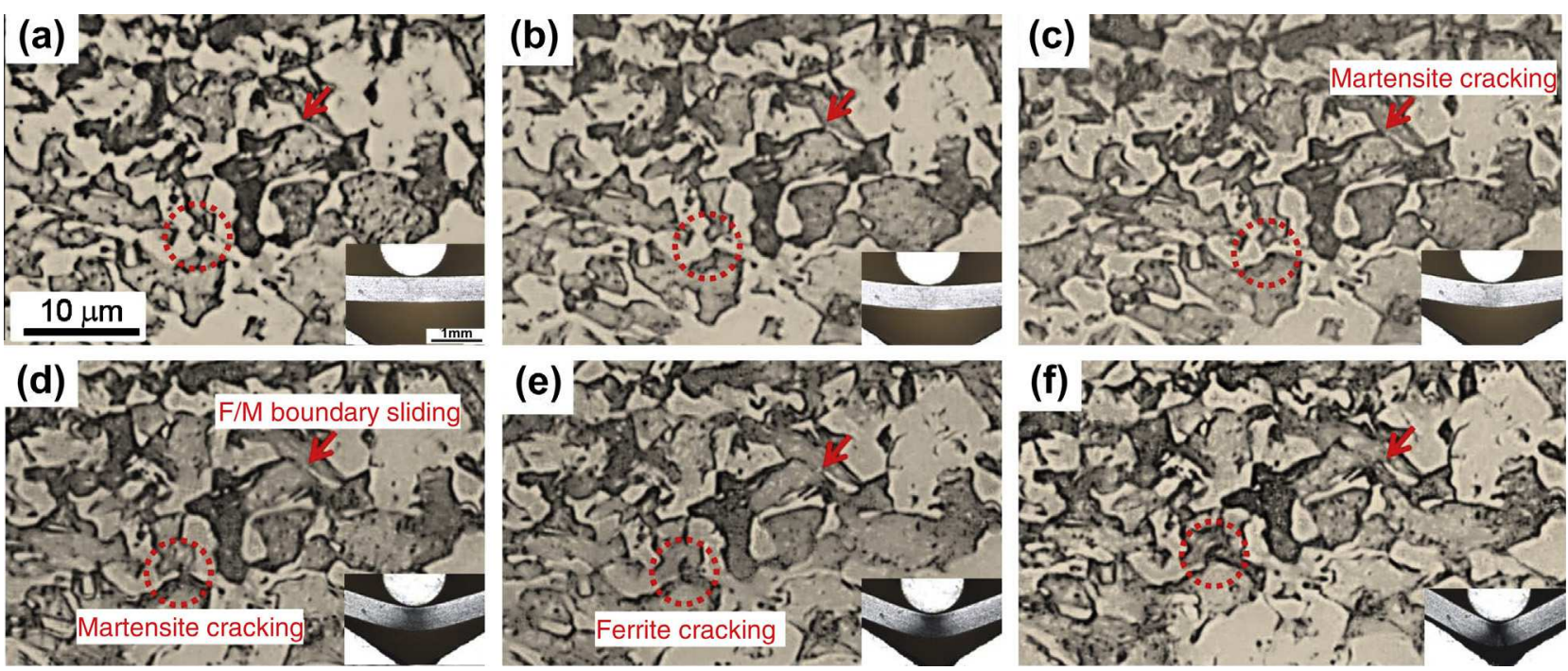

Fig. 11. In situ light microscopic observations during bending after hydrogen precharging. The corresponding video is attached as a Supplementary video file. The red arrow and dotted circle indicate the same position among the micrographs. The lower right images in each figure show a low-magnification image of the three-point bending test stage. Martensite is the darker phase, as a result of etching. The red arrows indicate the same edge of martensite. All images were taken in the tensile deformed lower region of the specimen. (For interpretation of the references to color in this figure legend, the reader is referred to the web version of this article.)

HEDE-related mechanism, reducing the damage incubation regime in Fig. 4c.

After a crack is arrested at the surrounding ferrite grain, the further propagation of this crack is controlled by two phenomena, namely, ferrite/martensite interface cracking and the cracking of the ferrite grain itself (as shown in Figs. 5 and $8 \mathrm{f}$ and g). The former is again a HEDE-based process, and the latter is explained within the framework of the HELP theory. Apparently, the HEDE and HELP mechanisms act here in a cooperative manner, reducing the damage nucleation regime and increasing the crack growth rate (Fig. 4c). In particular, the HELP effect contributes to the reduction in the critical strain, since the well-grown brittle fracture surface shown in Fig. 7b includes a considerable number of voids and dimples which are characteristic features of ductile fracture. Note that such hydrogen-assisted crack propagation phenomena have been often observed in iron and steels [62-65]. Also, in a ferrite/pearlite dual-phase steel, the hydrogen-assisted ductile crack propagation to ferrite has been reported to stem from the hydrogen localization at the tip of a brittle crack in pearlite [65].

The relative importance of the HEDE and HELP mechanisms can be clarified through the interrupted test results presented in Fig. 2. Since 5-6\% plastic strain corresponds to the uniform deformation (see Fig. 3c), the interruption of the tensile test of the hydrogen-precharged sample at $6 \%$ strain represents a prestraining just up to necking. One should note that the $6 \%$ macroscopic strain corresponds here to almost the same level of local plastic strain. This strain level corresponds to the damage nucleation regime marked in Fig. 4c. In this regime martensite cracking has already been initiated, but no crack growth into ferrite has yet occurred when the tensile test was interrupted. Since most of the diffusible hydrogen was desorbed after an exposure to air for 10 days, no direct hydrogen effect should be present when the tensile test is restarted after the interruption. Therefore, as seen in Fig. 7c, the size of the brittle feature region indicated by the yellow arrow is much larger than the grain size, but smaller than that in the fully hydrogen-affected specimen. This observation indicates that the reduction in elongation compared to the test without hydrogen charging is caused by martensite cracking associated with HEDE. Since the average size of HEDE induced-cracks stayed fixed in the damage nucleation regime (i.e. due to the crack arrest at surrounding ferrite), martensite cracking is considered to promote coalescence of voids and cracks due to the increased number of microcracks, accelerating the final catastrophic failure. When compared to the test conducted with hydrogen precharging, a recovery of the elongation is observed due to hydrogen desorption, indicating that the HEDEand HELP-assisted crack propagation which occurred in the crack growth regime also plays a key role in the hydrogen-induced degradation of the tensile property. Hence, the interrupted test provides evidence that HE of DP steels is caused by the combined effect of HEDE and HELP.

These results are also interesting from a microstructure design point of view, as they indicate that improvement in the HE of DP steels requires ways not only to improve HEDE-induced martensite cracking behavior (e.g. by employing tempering treatments), but also to retrieve HELP-induced loss in ferrite crack-arresting ability (e.g. by employing compositions that lead to a higher strain hardening capability of the ferrite phase). 


\section{Conclusions}

The microcracking phenomena in martensite-ferrite dual-phase steels were investigated in the presence of hydrogen. We used a quantitative, micromechanical and microstructure-sensitive approach. The results show that the damage evolution process in DP steels can be generally understood in terms of three basic regimes, namely, damage incubation, damage nucleation and damage growth. We observe that hydrogen charging strongly affects all of them. The first regime is characterized by crack incubation, i.e. when a critical plastic strain is reached decohesionbased martensite cracking is initiated (damage incubation regime). In the second regime, once this critical strain is reached, damage sets in, with a constant average crack size due to the ferrite phase arresting these cracks (crack nucleation regime). The third regime is characterized by the fact that, upon further straining, a critical strain for the onset of crack growth is reached, and crack propagation and opening take place, increasing the average crack size until occurrence of final fracture (crack growth regime). Hydrogen not only decreases the critical strain for the decohesion in martensite regions through the HEDE mechanism, but also promotes ferrite/martensite cracking, ferrite/martensite boundary sliding and cracking of the ferrite grains. While the ferrite/martensite cracking is also attributed to decohesion, the ferrite/martensite boundary sliding and ferrite cracking mechanisms are associated with enhanced dislocation mobility, i.e. the HELP mechanism. Thus, in the presence of hydrogen, both HEDE and HELP mechanisms assist the damage evolution in dual-phase steels in a cooperative manner, causing macroscopic hydrogen embrittlement. Consequently, higher resistance against hydrogen embrittlement of these materials can only be achieved by simultaneously reducing both of these effects.

\section{Acknowledgements}

The authors gratefully acknowledge the financial support by the Elements Strategy Initiative for Structural Materials (ESISM) through the Ministry of Education, Culture, Sports, Science and Technology (MEXT) of Japan. M.K. acknowledges the Research Fellowship of the Japan Society for the Promotion of Science for Young Scientists. We also acknowledge Mr. Yuki Toji, JFE Steel, for taking part in the discussions during the experiments.

\section{Appendix A. Supplementary material}

Supplementary data associated with this article can be found, in the online version, at http://dx.doi.org/10.1016/ j.actamat.2014.01.048.

\section{References}

[1] Toji Y, Takagi S, Yoshino M, Hasegawa K, Tanaka Y. Mater Sci Forum 2010;638-642:3537.
[2] Calcagnotto M, Ponge D, Raabe D. Metall Mater Trans A 2012;43A:37.

[3] Peranio N, Li YJ, Roters F, Raabe D. Mater Sci Eng A 2010:527:4161.

[4] Calcagnotto M, Ponge D, Raabe D. Mater Sci Eng A 2010;572:7832.

[5] Calcagnotto M, Adachi Y, Ponge D, Raabe D. Acta Mater 2011;59:658.

[6] Chang P-H, Preban AG. Acta Mater 1985;33:897.

[7] Sun X, Choi KS, Liu WN, Khaleel MA. Int J Plasticity 2009;25:1888.

[8] Morooka S, Umezawa O, Harjo S, Hasegawa K, Yoji Y. Tetsu-toHagané 2012;98:311.

[9] Kadkhodapour J, Schmauder S, Raabe D, Ziaei-Rad S, Weber U, Calcagnotto. Acta Mater 2011;59:4387.

[10] Tasan CC, Hoefnagels JPM, Geers MGD. Scripta Mater 2010;62:835.

[11] Ghadbeigi H, Pinna C, Celotto S, Yates JR. Mater Sci Eng A 2010;527:5026.

[12] Minami H, Nakayama K, Morikawa T, Higashida K, Toji Y, Hasegawa K. Tetsu-to-Hagané 2011;97:493.

[13] Johnson HH, Troiano AR. Nature 1957;179:777.

[14] Johnson HH. In: Gibala R, Hehemann RF, editors. Hydrogen embrittlement and stress corrosion cracking. Materials Park, OH: ASM International; 1984.

[15] Cotterill P. Prog Mater Sci 1961;9:205.

[16] Davies RG. Metall Trans A 1981;12A:1667.

[17] Davies RG. Scripta Metall 1983;17:889.

[18] Sun S, Gu J, Chen N. Scripta Metall 1989;1735:1737.

[19] Pérez Escobar D, Miñambres C, Duprez L, Verbeken K, Verhaege M. Corros Sci 2011;53:3166.

[20] Frohmberg RP, Barnett WJ, Troiano AR. WADC tech rep 1954:54. $<$ http://contrails.iit.edu/DigitalCollection/1954/WADCTR54-320.pdf>.

[21] Perng TP, Altstetter CJ. Metall Trans A 1987;18A:123.

[22] Nagumo M, Yagi T, Saitoh H. Acta Mater 2000;48:943.

[23] Troiano AR. Trans ASM 1960;52:54.

[24] Oriani RA. Ann Rev Mater Sci 1978;8:327.

[25] Hirth JP. Metal Trans A 1980;11A:861.

[26] Birnbaum HK, Sofronis P. Mater Sci Eng A 1994;176:191.

[27] Robertson IM, Birnbaum HK. Acta Metall 1986;34:353.

[28] von Pezold J, Lymperakis L, Neugebauer J. Acta Mater 2011;59:2969.

[29] Maxelon JM, Pundt A, Pyckhout-Hintzen W, Barker J, Kirchheim R. Acta Mater 2001;49:2625.

[30] Neeraj T, Srinivasan R, Li J. Acta Mater 2012;60:5160.

[31] Nagao A, Smith CD, Dadfarnia M, Sofronis P, Robertson IM. Acta Mater 2012;60:5182.

[32] Wang M, Akiyama E, Tsuzaki K. Corros Sci 2007;49:4081.

[33] Lovicu G, Bottazzi M, D'aiuto F, De Sanctis M, Dimatteo A, Santus C, et al. Metall Mater Trans A 2012;43A:4075.

[34] Shibata A, Takahashi H, Tsuji N. ISIJ Int 2012;52:208.

[35] Takagi S, Toji Y, Yoshino M, Hasegawa K. ISIJ Int 2012;52:316.

[36] Enos DG, Scully JR. Metall Mater Trans A 2002;33A:1151.

[37] Lemaitre J, Dufailly J. Eng Fract Mech 1987;28:643.

[38] Tasan CC, Hoefnagels JPM, Geers MGD. Acta Mater 2012;60: 3581.

[39] Tasan CC. PhD thesis, Eindhoven University of Technology. <http:// www/mate.tue.nltohttp://www.mate.tue.nl>.

[40] Gutierrez-Urrutia I, Zaefferer S, Raabe D. Scripta Mater 2009;61:737.

[41] Koyama M, Akiyama E, Sawaguchi T, Raabe D, Tsuzaki K. Scripta Mater 2012;66:459.

[42] Koyama M, Akiyama E, Tsuzaki K, Raabe D. Acta Mater 2013;61:4607.

[43] Koyama M, Akiyama E, Sawaguchi T, Ogawa K, Kireeva IV, Chumlyakov YI, et al. Corros Sci 2013;75:345.

[44] Forcey KS, Ross DK, Simpson JCB. J Nucl Mater 1988;160:117.

[45] Luu WC, Wu JK. Corros Sci 1996;239:245.

[46] Parvathavarthini N, Saroja S, Dayal RK. J Nucl Mater 1999;264:35.

[47] Takai K, Watanuki R. ISIJ int 2003;43:520.

[48] Pérez Escobar D, Depover T, Duprez L, Verbeken K, Verhaege M. Acta Mater 2012;60:2593. 
[49] Akiyama E, Matsukado K, Wang M, Tsuzaki K. Corros Sci 2010;52:2758.

[50] Scully JR, Dogan H, Li D, Gangloff RP. Proc Conf Corros 2004:04563.

[51] Raabe D, Sachtleber M, Zhao Z, Roters F, Zaefferer S. Acta Mater 2001;49:3433.

[52] Zhao Z, Ramesh M, Raabe D, Cuitino A, Radovizky R. Int J Plasticity 2008;24:2278.

[53] Brewer LN, Field DP, Merriman CC. In: Schwartz AJ, Kumar M, Adams BL, Field DP, editors. Electron backscatter diffraction in material science. New York: Springer Science + Business Media; 2000.

[54] Demir E, Raabe D, Zaafarani N, Zaefferer S. Acta Mater $2009 ; 57: 559$
[55] Calcagnotto M, Ponge D, Demir E, Raabe D. Mater Sci Eng A 2010;527:2738.

[56] Choo WY, Lee JY. Metall Trans A 1982;13A:135.

[57] Chan SLI. J Chi Inst Eng 1999;22:43.

[58] Tasan CC, Hoefnagels JPM, ten Horn CHLJ, Geers MGD. Mech Mater 2009;41:1264.

[59] Morito S, Tanaka H, Konishi R, Furuhara T, Maki T. Acta Mater 2003;51:1789.

[60] Takai K, Seki J, Homma Y. Mater Trans JIM 1955;36:1134.

[61] Kim YH, Morris Jr JW. Metall Trans A 1983;14A:1883.

[62] Gao M, Wie RP. Acta Metall 1984;32:2115.

[63] Tabata T, Birnbaum HK. Scripta Metall 1984;18:231.

[64] Beachem CD. Metall Trans 1972;3:437.

[65] Nishiguchi H, Fukushima Y, Matsuoka S, Murakami Y. Trans Jpn Soc Mech Eng A 2010;76:1459. 